\title{
Counting of finite topologies and a dissection of Stirling numbers of the second kind
}

\section{Krishnamurthy}

\begin{abstract}
Certain new combinatorial numbers which arise in the counting of finite topologies are introduced and formulae obtained. These numbers are used to obtain a known formula for $t_{n}$, the number of labelled topologies on $n$ points in terms of the Stirling numbers $S(n, p)$ and $d_{n}$, the number of labelled $T_{0}$-topologies on $n$ points. The numbers $d_{n}$ are computed for $n \leq 5$ with the the help of a method of Comtet (1966) (which seems to have been missed by later authors), reinterpreted for transitive digraphs.
\end{abstract}

\section{Introduction}

Let $X=\{1,2, \ldots, n\}$. Let $t_{n}$ (and $d_{n}$ ) stand for the number of labelled topologies (and labelled $T_{0}$-topologies) respectively, on $X$. That

$$
t_{n}=\sum_{p} s(n, p) d_{p}
$$

is well known (ef. Evans, Harary and Lynn, [2], Comtet, [1], Gupta, [3]) and implicit in Shafaat, [6]. Comtet, [1], also derived a formula for the calculation of $d_{p}$ and Shafaat, [6], has a similar formula.

Received 23 August 1974. Propositions 1 and 2 and Theorem 1 were done, a few years ago, jointly with M.S. Radhakrishnan and S. Raghunath. Prublieation of this paper was delayed to enable the author to make a minor correction to Example 1. Editor\} 
In this paper we introduce certain combinatorial numbers, $\lambda(n: r: p)$, which arise in the counting of finite topologies on $X$. These numbers satisfy

$$
\sum_{r} \lambda(n: r: p)=S(n, p)
$$

We prove, independently of (1), that

$$
t_{n}=\sum_{p} \sum_{r} \lambda(n: r: p) d_{p}
$$

We then take up the calculation of $d_{p}$ and provide, via transitive digraphs, what seems to be an easier version of Comtet's formula. Our computed values of $\lambda(n: r: p)$ and of $d_{n}, n \leq 5$, lead to known values of $t_{n}$ as given in Comtet, [1], and Evans, Harary and Lynn, [2]. Shafaat's method [6], which is akin to that of Comtet, ends up in results for $t_{5}$ and $t_{6}$ that are wrong.

Unless otherwise mentioned all our topologies and graphs are labelled.

\section{Combinatorial numbers $\lambda(n: r: p)$ and proof of (3)}

We start with the concept of 'Borel equivalence' introduced by Rayburn [5]. Let $T(X)$ be the set of all topologies on $X$. Two topologies on $X$ are said to be Borel equivalent if they generate the same Borel field; that is, a topology in which every open set is also closed, or, what Sharp, [7], calls, a symmetric topology. This equivalence partitions $T(X)$ into what are called Borel equivalence classes. 'How many topologies are there in each Borel equivalence class?' was a question posed by Rayburn.

Recall [2] that $T(X)$ is in one-to-one correspondence with transitive digraphs (shortly, transgraphs) in the following manner. Given $\tau \in T(X)$, denote by $B_{i}$ the smallest $\tau$-open set containing $i$. Construct the directed graph $G(\tau)$ on $X$ by stipulating that, for $j \neq i$, $(i, j) \in G(\tau)$ if and only if $j \in B_{i}$. (Here, and throughout the paper, $(i, j)$ means the directed edge leading from $i$ to $j$.) The fact that this construction results in $G(\tau)$ being transitive and that the correspondence $\tau \rightarrow G(\tau)$ is bijective are proved in [2] and [4]. Under 
this correspondence, $T_{0}$-topologies and Borel fields show themselves up as two extremes in $T(X)$. Let us use the term 'dwicycle' to denote a directed cycle of length two.

Then $T_{0}$-topologies correspond to transgraphs which have no dwicycles (cf. [2] and [7]) and Borel fields correspond to transgraphs in which every edge is part of a dwicycle. These can be seen easily by noting that:

(1) $\tau$ is a $T_{0}$-topology if and only if $j \in B_{i} \Rightarrow i \mid B_{j}$; and

(2) $\tau$ is a Borel field if and only if $j \in B_{i} \Rightarrow i \in B_{j}$ (cf. Rayburn [5]).

Also note that $\tau_{1}$ is finer than $\tau_{2}$ if and only if $G\left(\tau_{1}\right)$ is a subgraph of $G\left(\tau_{2}\right)$. This tells us that, to generate the Borel field $B_{\tau}$ containing $\tau$, we have only to look at the subgraph of $G(\tau)$ and pick the largest subgraph $G_{0}$ which has nothing but dwicycles in it. This $G_{0}$ will be $G\left(B_{\tau}\right)$. We have thus proved

PROPOSITION 1. If $\tau \in T(X)$ and $B_{\tau}$ is the Borel field generated by $\tau$ then $G\left(B_{\tau}\right)$ can be obtained from $G(\tau)$ by deleting all the edges in the latter which are not part of dwicycles.

As an illustration, note the following transgraphs on three points:

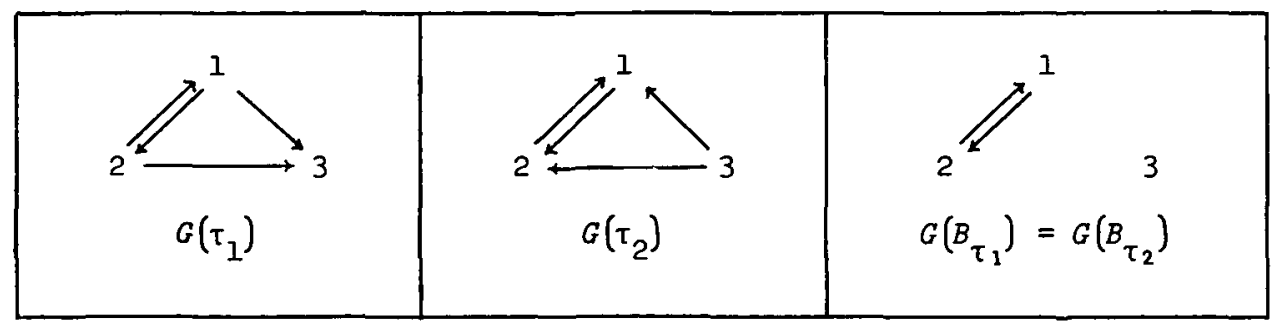

If $\tau$ is in the Borel equivalence class $B(B)$ determined by $B$ then $G(\tau)$ and $G(B)$ differ only in the single lines which do not form part of dwicycles. To construct $G(\tau)$ from $G(B)$, we have, therefore, only to add other lines to $G(B)$ in such a way that

(i) the resulting graph is a transgraph, and 
(ii) no new dwicycles are introduced.

Note that, in this construction, if $(i, j)$ and $(j, i)$ form a dwicycle and $k$ is any other vertex, either we add both $(i, k)$ and $(j, k)$ or not at all and similarly, either we add both $(k, i)$ and $(k, j)$ or not at all. So, for the purpose of this construction, we can identify pairs of points which are connected by a dwicycle. Consider the resulting smaller set $X_{0}$ of points and construct transgraphs on $X_{0}$ without dwicycles. For each such transgraph on $X_{0}$ (which is now a $T_{0}$-topology on $X_{0}$ ) we can recover a topology on $X$ which belongs to $B(B)$. This is done by recovering all the identified points and the dwicycles connecting them. Conversely every $T_{0}$-topology on $X_{0}$ in the same way gives rise to a topology on $X$ which belongs to $B(B)$. Thus the number of topologies in $B(B)$ is the number of $T_{0}$-topologies on the set $X_{0}$ as obtained above. Hence we have proved the following

PROPOSITION 2. Let $B$ be a Borel fiezd and $C(G(B))$ be the groph obtained by identifying pairs of points in $G(B)$ which are connected by dwicycles. Let $p$ be the number of vertices in $C(G(B))$. Then the number of topologies in the Borel equivalence class determined by $B$ is $d_{p}$

Now in order to count $|T(X)|$ we have only to list the various Borel equivalence classes there are and sum up $d_{p}$ for the various values of $p$ that arise. But it happens that the same $p$ may arise from distinct Borel fields, as can be seen from the two transgraphs on four points shown below.

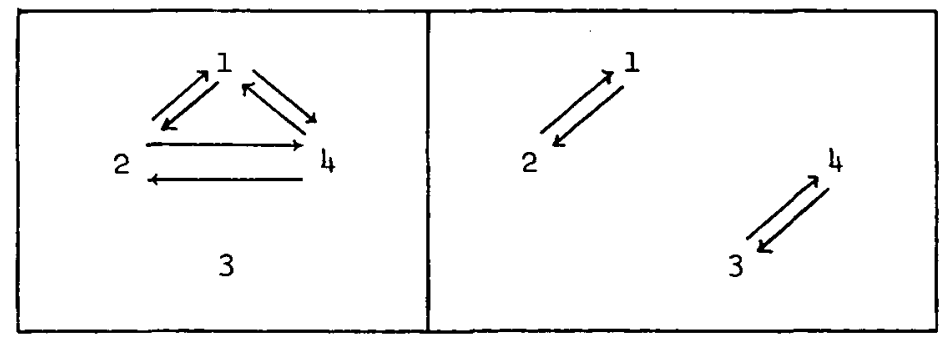

So it is necessary to take into account the number $r$ of dwicycles that occur in $G(B)$. Each unlabelled Borel field $B$ is determined by two parameters $r$ and $p$. So we make the following definition. 
DEFINITION 1. Given integers $n, r, p$ such that $n \geq 2$, $0 \leq r \leq\left(\begin{array}{l}n \\ 2\end{array}\right)$ and $1 \leq p \leq n, \lambda(n: r: p)$ denotes the number of labelled Borel fields $B$ on a set of $n$ points, with $r$ dwicycles and with $p=|C(G(B))|$. If there is no such Borel field for a pair $\left(r_{0}, p_{0}\right)$, $\lambda\left(n: r_{0}: p_{0}\right)=0$.

Putting aside the calculation of $\lambda(n: r: p)$ for a while, we first note that Proposition 2 and the discussion following it gives us the following

THEOREM 1. $t_{n}=\sum_{p} \sum_{r} \lambda(n: r: p) d_{p}$

Recall ( $c f$. Sharp [7]) that $T(X)$ is in bijective correspondence with the set of quasiorders (reflexive and transitive relations) on $X$, by the rule

$$
j \in B_{i} \Leftrightarrow i R j
$$

Under this correspondence $T_{0}$-topologies correspond to partial orders and Borel fields correspond to equivalence relations. Given $p$, the problem of constructing all the $\sum_{r} \lambda(n: r: p)$ labelled Borel fields is the problem of distributing $n$ distinct objects (the vertices $1,2, \ldots, n$ in this case) into $p$ distinct cells (the vertices of $C(G(B)$ ), in this case). Hence

$$
\sum_{r} \lambda(n: r: p)=S(n, p)
$$

This observation completes the promised independent proof of (1).

\section{Calculation of $\lambda(n: r: p)$}

When $r=0, p=n$, and clearly $\lambda(n: 0: n)=1$. We shall suppose $r>0$ in the rest of this section until we come to Theorem 2 . The number $r$ arises as follows. First, note that, as a consequence of transitivity, no dwicycle can exist in a transgraph except as part of a complete sub-transgraph. The number $r$ will therefore be the sum of the numbers of dwicycles in the complete subtransgraphs of $G(B)$. But the 
number of dwicycles in a complete subtransgraph is $\left(\begin{array}{l}k \\ 2\end{array}\right)$ where $k \geq 2$ is the number of vertices in the complete subtransgraph. So,

$$
r=\left(\begin{array}{c}
k_{1} \\
2
\end{array}\right)+\left(\begin{array}{c}
k_{2} \\
2
\end{array}\right)+\ldots
$$

with $k_{i} \geq 2$, and $k_{1}+k_{2}+\ldots=n$. The number $p$ is the number of such complete subtransgraphs in $G(B)$. Thus, given the parameters $n, r, p$ we arrive at a unique unordered partition of the integer $n$ into $p$ parts such that $n=k_{1}+k_{2}+\ldots+k_{p}$ and $r=\sum_{i=1}^{p}\left(\begin{array}{c}k_{i} \\ 2\end{array}\right)$.

Conversely, given an unordered partition of the integer $n$ which has at least one part greater than 1 , the parameters $r$ and $p$ are determined uniquely.

Thus this correspondence between unordered partitions with at least one part greater than $I$ and the triads of parameters $n, r, p$ for which $\lambda(n: r: p)>0$ is bijective. So, to determine $\lambda(n: r: p)$, we take the corresponding partition

$$
n=k_{1}+k_{2}+\ldots+k_{p}
$$

and regroup the integers $k_{1}, k_{2}, \ldots, k_{p}$ into

$$
\begin{aligned}
& \alpha_{1} \text { integers each equal to } p_{1}, \\
& \alpha_{2} \text { integers each equal to } p_{2} \text {, and so on. }
\end{aligned}
$$

(Note that we must have $\sum \alpha_{i} p_{i}=n$ and at least one $p_{i} \geq 2$.) The corresponding tranggraph will consist of

$\alpha_{1}$ disfoint complete transgraphs each on $p_{1}$ points;

$\alpha_{2}$ disjoint complete transgraphs each on $p_{2}$ points; and so on; subject to the understanding that wherever $p_{j}=1$, the component corresponding to that reduces to a single point. "In how many ways can such a configuration arise, with $n, r, p$ given?" is the question. The choice of $\alpha_{1}$ subsets of $p_{1}$ vertices each can be made in 


$$
\frac{\left(\begin{array}{l}
n \\
p_{1}
\end{array}\right)\left(\begin{array}{c}
n-p_{1} \\
p_{1}
\end{array}\right)\left(\begin{array}{c}
n-2 p_{1} \\
p_{1}
\end{array}\right) \ldots\left(\begin{array}{c}
n-\left(\alpha_{1}-1\right) p_{1} \\
p_{1}
\end{array}\right)}{\alpha_{1} !}
$$

ways. Having made this choice, the choice of $\alpha_{2}$ subsets of $p_{2}$ vertices each can be made in

$$
\frac{\left(\begin{array}{c}
n-\alpha_{1} p_{1} \\
p_{2}
\end{array}\right)\left(\begin{array}{c}
n-\alpha_{1} p_{1}-p_{2} \\
p_{2}
\end{array}\right) \cdots\left(\begin{array}{c}
n-\alpha_{1} p_{1}-\left(\alpha_{2}-1\right) p_{2} \\
p_{2}
\end{array}\right)}{\alpha_{2} !}
$$

ways; and so on.

This completes the proof of the following

THEOREM 2. Let $n$ be cony integer greater thon or equal to 2 , $0 \leq r \leq\left(\begin{array}{l}n \\ 2\end{array}\right)$ and $1 \leq p \leq n$ such that $n=k_{1}+k_{2}+\ldots+k_{p}$ and $r=\sum_{i}\left(\begin{array}{c}k_{i} \\ 2\end{array}\right)$ where $\left(\begin{array}{c}k_{i} \\ 2\end{array}\right)=0$ if $k_{i}=1$. Then

$\lambda(n: r: p)=\frac{\left(\begin{array}{l}n \\ p_{1}\end{array}\right)\left(\begin{array}{c}n-p_{1} \\ p_{1}\end{array}\right)\left(\begin{array}{c}n-2 p_{1} \\ p_{1}\end{array}\right) \cdots\left(\begin{array}{c}n-\left(\alpha_{1}-1\right) p_{1} \\ p_{1}\end{array}\right)}{\alpha_{1} !}$

$$
\times \frac{\left(\begin{array}{c}
n-\alpha_{1} p_{1} \\
p_{2}
\end{array}\right)\left(\begin{array}{c}
n-\alpha_{1} p_{1}-p_{2} \\
p_{2}
\end{array}\right) \ldots\left(\begin{array}{c}
n-\alpha_{1} p_{1}-\left(\alpha_{2}-1\right) p_{2} \\
p_{2}
\end{array}\right)}{\alpha_{2} !} \times \ldots
$$

where the integers $k_{1}, k_{2}, \ldots, k_{p}$ have

$$
\begin{aligned}
& \alpha_{1} \text { integers each equal to } p_{1}, \\
& \alpha_{2} \text { integers each equal to } p_{2} \text {, and so on. }
\end{aligned}
$$

\section{Calculation of $d_{p}$}

It remains to calculate $d_{p}$ for every $p>0$. clearly $d_{1}=1$ and $d_{2}=3$. To arrive at a general formula for $d_{p}$, we proceed by the method of Comtet but now use the concept of transgraphs intensively. Evans, 
Harary and Lynn [2] have done a similar computation but ours is different.

Let $\Gamma_{n}$ be the set of transgraphs on $n$ points without dwicycles. Let $\gamma$ stand for an arbitrary element of $\Gamma_{n}$. We shall associate with each $\gamma$ a unique ordered vector of non-empty subsets of $X$ as follows. Count the outdegrees of each vertex of $\gamma$. (The outdegree of a vertex is the number of directed edges leaving it.) We claim that at least one of these outdegrees must be zero. To see this, start with any vertex $i \in \gamma$. If $j \in B_{i}$, then $(i, j) \in \gamma$ but $(j, i) \notin \gamma$. Now look at $B_{j}$. If $k \in B_{j}$ then $k$ can be connected only to points other than $i$ and $j$; this follows easily from the transitivity of $\gamma$ and the fact that it has no dwicycles. Continuing this process, we finally end up with a vertex $p$ which is not connected to any other vertex. Thus there exists a $p$ such that the outdegree of $p$ is zero. Let $S_{1}(\gamma)$ be the set of all vertices of $\gamma$ with outdegree zero.

Delete all vertices belonging to $S_{1}(\gamma)$ from the graph $\gamma$ and also all the edges leading from or to such vertices. The resulting graph may be called the first truncation $\gamma_{1}$. Clearly it is a transgraph without dwicycles. Compute $S_{1}\left(\gamma_{1}\right)$ and denote it by $S_{2}(\gamma)$. Delete from $\gamma_{1}$, the points of $S_{2}(\gamma)$ and all edges leading from or to them, thus obtaining the second truncation $\gamma_{2}$. Continue this process until all vertices of $\gamma$ are exhausted. The last set $S_{k}(\gamma)$ will be such that all its points have outdegrees zero in the $(k-I)$ th truncation of $\gamma$. Write

$$
(s)_{\gamma}=\left(s_{1}(\gamma), s_{2}(\gamma), \ldots, s_{k}(\gamma)\right) \text {. }
$$

Thus, corresponding to $\gamma$ we have an ordered partition of non-empty subsets of $X$. We write $n(\gamma)=(S)_{\gamma}$. Note that $n$ of the discrete graph is $(X)$.

PROPOSITION 3. (i) $n$ is onto the set of all ordered partitions of non-empty subsets of $X$.

(ii) $n$ is many-one.

Proof. (i) Given an ordered partition $(s)=\left(s_{1}, s_{2}, \ldots, s_{k}\right)$ of 
non-empty subsets of $X$, we produce a transgraph $\gamma$ as follows. A directed edge goes from every point of $S_{p}$ to one or more points of $S_{q}$, $q<p$, in such a way that, whenever $p>2, x \in S_{p}, y \in S_{p-1}$, and $(x, y) \in \gamma$, it is true that, for every $i \leq p-2$,

$$
z \in S_{i} \text { and }(y, z) \in \gamma \Rightarrow(x, z) \in \gamma \text {. }
$$

The resulting $\gamma$ is clearly transitive. It has no dwicycles because all directed edges go from points of $S_{i}$ to points of $S_{j}, i>j$, and never in the opposite direction. The points of $S_{1}$ are all of outdegree zero. So $S_{1}=S_{1}(\gamma)$. $\gamma_{1}=\gamma S_{1}(\gamma)$, the first truncation of $\gamma$, has the points of $S_{2}$ as its set of vertices with zero outdegree. Hence $S_{2}=S_{2}(\gamma)$; and so on. Thus $n(\gamma)=(S)$ and $(i)$ is proved.

(ii) To prove (ii) look at $X=\{1,2,3\}$. Suppose $(S)=(\{1,3\},\{2\})$. Then all the following 3 -transgraphs have $(S)$ as their n-image.

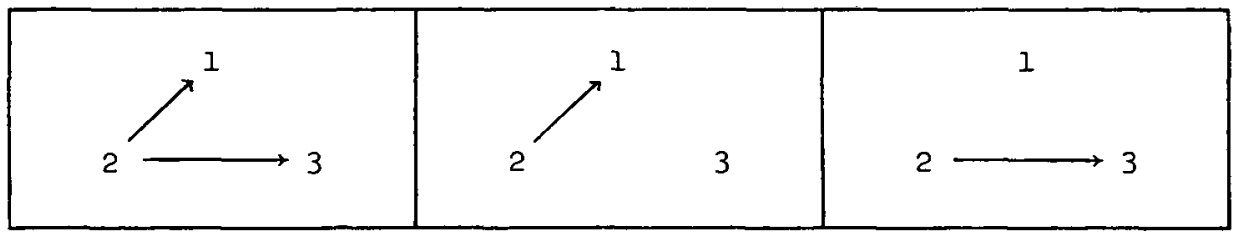

Let $N(S)$ be the number of $\gamma \in \Gamma_{n}$ such that $\eta(\gamma)=(S) \cdot N(S)$ can be computed for each $(S)$ (see Section 4). Given $(S)=\left(S_{1}, S_{2}, \ldots, S_{k}\right)$ where $\left|S_{i}\right|=s_{i}$, the number of ways in which the $n$ labelled vertices of $\gamma$ can be distributed into $s_{1}, s_{2}, \ldots, s_{k}$ is $\frac{n !}{s_{1} ! s_{2} ! \cdots s_{k} !}$. This proves the theorem of Comtet [1] as stated below.

THEOREM 3.

$$
\begin{aligned}
& d_{n}=\sum_{1 \leq k \leq n}(S)=\left(S_{1}, S_{2}, \ldots, s_{k}\right)^{\frac{n !}{s_{1} ! 8_{2} ! \cdots s_{k} !}} N(S) \\
& \left|s_{i}\right|=s_{i}>0, s_{i} \subset X \\
& \sum s_{i}=n
\end{aligned}
$$


where $N(S)$ is the number of transgraphs $Y$ on $X$, without diricycles such that $n(\gamma)=(S)$.

\section{Computation of $d_{n}, \lambda(n: s: p)$, and $t_{n}$}

NOTE. In this section and in the Tables at the end, we use (xyz) for $\{x, y, z\}$.

In the computation of $d_{n}$ the main problem is to calculate $N(S)$ for each possible form of $(s)=\left(s_{1}, s_{2}, \ldots, s_{k}\right)$ where $\left(\left|s_{1}\right|,\left|s_{2}\right|, \ldots,\left|s_{k}\right|\right)$ is an ordered partition of the integer $n$. Given $(S)$ we proceed as follows. For each point $x$ of $S_{p}$ and every $q<p$ we have to choose a point or points of $S_{q}$ to which lines from $x$ will lead. In other words, for each $x \in S_{p}$, one has to choose a non-empty subset of $s_{q}$. It helps to write all the possible choices for all $p$ and $q, p>q$, in the form of a tableau as below with $k-1$ rows and $k-1$ columns where the square at the row titled $S_{p}$ and the column titled $S_{q}$ lists all the choices for the map $S_{p} \rightarrow$ set of non-empty subsets of $S_{q}$. Then a case by case checking is done for transitivity.

Let us illustrate this with two examples.

EXAMPLE 1. $n=5,(S)=(12)(3)(4)(5)$.

\begin{tabular}{|c|c|c|}
\hline $3 \stackrel{(1)}{\longrightarrow}\left(\begin{array}{l}(1) \\
(12)\end{array}\right.$ & & \\
\hline $4 \underset{(12)}{\longrightarrow}\left(\begin{array}{l}(1) \\
(2)\end{array}\right.$ & $4 \rightarrow(3)$ & \\
\hline $5 \stackrel{x}{\longrightarrow_{(12)}}\left(\begin{array}{l}(1) \\
(2)\end{array}\right.$ & $5+(3)$ & $5 \rightarrow(4)$ \\
\hline
\end{tabular}


The choice $3 \rightarrow(1)$ implies $4 \rightarrow(1)$ and $5+(1)$ (12) or $4 \rightarrow$ (12) and $5 \rightarrow(12)$. This gives 3 choices with $3 \rightarrow(1)$. Similarly there are 3 choices with $3 \rightarrow(2)$. The choice $3 \rightarrow(12)$ implies $4 \rightarrow(12)$ and $5 \rightarrow(12)$. Thus $N(S)=3+3+1=7$.

EXAMPLE 2. $n=5,(s)=(12)(34)(5)$.

(34)

(5)

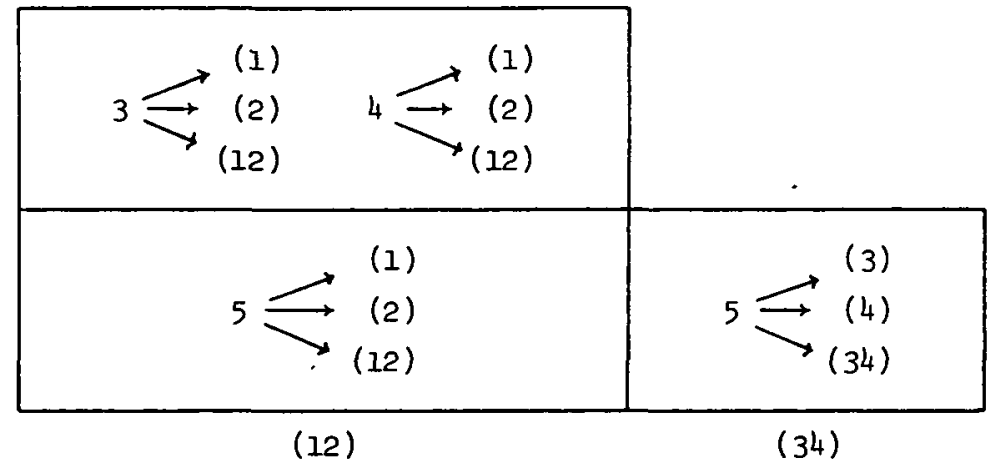

Start with $5 \rightarrow(3)$ and $3 \rightarrow(1)$. This goes with any choice for the image of 4 and implies $5 \underset{(12)}{\longrightarrow}$. Hence there are 6 choices.

Similarly there are 6 choices with $5+(3)$ and $3 \rightarrow(2)$. There are only 3 choices that go with $5 \rightarrow(3)$ and $3 \rightarrow(12)$. Thus there are, in all, 15 choices for $5 \rightarrow(3)$. Similarly there are 15 choices for $5 \rightarrow(4)$.

$$
\begin{aligned}
& \text { Now take up } 5 \rightarrow(34) \text {. Then } \\
& 3+(1), 4+(1) \Rightarrow 5>(1), \\
& 3+(1),, 4+(2) \quad \Rightarrow 5 \rightarrow(12) \text {, } \\
& 3 \rightarrow(1), 4+(12) \Rightarrow 5+(12) \text {. }
\end{aligned}
$$

Thus $3 \rightarrow(1)$ gives 4 choices. Similarly $3 \rightarrow(2)$ gives 4 choices. Finally $3 \rightarrow(12)$ gives 3 choices. Thus $5 \rightarrow(34)$ gives, in all, $4+4+3=11$ choices. Hence $N(S)=15+15+11=41$.

The completed results are tabulated in Table 1 for $2 \leq n \leq 5$. 
Table 2 gives the results for $\lambda(n: r: p)$ and the computations for obtaining $t_{n}, 2 \leq n \leq 5$.

Table 1

Computation of $d_{n}, 2 \leq n \leq 5$

\begin{tabular}{|c|c|c|c|c|c|c|}
\hline$n$ & $\begin{array}{l}\text { ordered } \\
\text { partition } \\
\text { of } n\end{array}$ & Typical form of $(S)$ & $N(S)$ & $\begin{array}{l}\text { Number of } \\
S^{\prime} \text { 's of the } \\
\text { same form }\end{array}$ & $\begin{array}{l}\text { Contribution } \\
\text { to } d_{n}\end{array}$ & $d_{n}$ \\
\hline 2 & $\begin{array}{r}2 \\
11\end{array}$ & $\begin{array}{l}(12) \\
(1)(2)\end{array}$ & $\begin{array}{l}1 \\
1\end{array}$ & $\begin{array}{l}1 \\
2\end{array}$ & $\begin{array}{l}1 \\
2\end{array}$ & 3 \\
\hline 3 & $\begin{array}{r}3 \\
12 \\
21 \\
111\end{array}$ & $\begin{array}{l}(123) \\
(1)(23) \\
(12)(3) \\
(1)(2)(3)\end{array}$ & $\begin{array}{l}1 \\
1 \\
3 \\
1\end{array}$ & $\begin{array}{l}1 \\
3 \\
3 \\
6\end{array}$ & $\begin{array}{l}1 \\
3 \\
9 \\
6\end{array}$ & 19 \\
\hline 4 & $\begin{array}{r}4 \\
13 \\
31 \\
22 \\
112 \\
121 \\
211 \\
1111\end{array}$ & $\begin{array}{l}(1234) \\
(1)(234) \\
(123)(4) \\
(12)(34) \\
(1)(2)(34) \\
(1)(23)(4) \\
(12)(3)(4) \\
(1)(2)(3)(4)\end{array}$ & $\begin{array}{l}1 \\
1 \\
7 \\
9 \\
1 \\
3 \\
5 \\
1\end{array}$ & $\begin{array}{r}1 \\
4 \\
4 \\
6 \\
12 \\
12 \\
12 \\
24\end{array}$ & $\begin{array}{l}1 \\
4 \\
28 \\
54 \\
12 \\
36 \\
60 \\
24\end{array}$ & 219 \\
\hline 5 & $\begin{array}{r}5 \\
14 \\
41 \\
23 \\
32 \\
221 \\
212 \\
122 \\
311 \\
131 \\
113 \\
1112 \\
1121 \\
1211 \\
2111 \\
11111\end{array}$ & $\begin{array}{l}(12345) \\
(1)(2345) \\
(1234)(5) \\
(12)(345) \\
(123)(45) \\
(12)(34)(5) \\
(12)(3)(45) \\
(1)(23)(45) \\
(123)(4)(5) \\
(1)(234)(5) \\
(1)(2)(345) \\
(1)(2)(3)(45) \\
(1)(2)(34)(5) \\
(1)(23)(4)(5) \\
(12)(3)(4)(5) \\
(1)(2)(3)(4)(5)\end{array}$ & $\begin{array}{r}1 \\
1 \\
15 \\
27 \\
49 \\
41 \\
9 \\
9 \\
19 \\
7 \\
1 \\
1 \\
3 \\
5 \\
7 \\
1\end{array}$ & $\begin{array}{r}1 \\
5 \\
5 \\
10 \\
10 \\
30 \\
30 \\
30 \\
20 \\
20 \\
20 \\
60 \\
60 \\
60 \\
60 \\
120\end{array}$ & $\begin{array}{r}1 \\
5 \\
75 \\
270 \\
490 \\
1230 \\
270 \\
270 \\
380 \\
140 \\
20 \\
60 \\
180 \\
300 \\
420 \\
120\end{array}$ & 4231 \\
\hline
\end{tabular}


Table 2

Values of $\lambda(n: r: p)$ and $t_{n}$

\begin{tabular}{|c|c|c|c|c|c|c|}
\hline$n$ & $\begin{array}{l}\text { Unordered } \\
\text { partition } \\
\text { of } n\end{array}$ & $\lambda(n: r: p)$ & $S(n, p)$ & $d_{p}$ & $\begin{array}{l}\text { Contribution } \\
\text { to } t_{n}\end{array}$ & $t_{n}$ \\
\hline 2 & $\begin{array}{r}11 \\
2\end{array}$ & $\begin{array}{l}\lambda(2: 0: 2)=1 \\
\lambda(2: 1: 1)=1\end{array}$ & $\begin{array}{l}S(2,2)=1 \\
S(2,1)=1\end{array}$ & $\begin{array}{l}3 \\
1\end{array}$ & $\begin{array}{l}3 \\
1\end{array}$ & 4 \\
\hline 3 & $\begin{array}{r}111 \\
21 \\
3\end{array}$ & $\begin{array}{l}\lambda(3: 0: 3)=1 \\
\lambda(3: 1: 2)=3 \\
\lambda(3: 3: 1)=1\end{array}$ & $\begin{array}{l}S(3,3)=1 \\
S(3,2)=3 \\
S(3,1)=1\end{array}$ & $\begin{array}{r}19 \\
3 \\
1\end{array}$ & $\begin{array}{r}19 \\
9 \\
1\end{array}$ & 29 \\
\hline 4 & $\begin{array}{r}1111 \\
211 \\
22 \\
31 \\
4\end{array}$ & $\left.\begin{array}{l}\lambda(4: 0: 4)=1 \\
\lambda(4: 1: 3)=6 \\
\lambda(4: 2: 2)=3 \\
\lambda(4: 3: 2)=4 \\
\lambda(4: 6: 1)=1\end{array}\right\}$ & $\begin{array}{l}S(4,4)=1 \\
S(4,3)=6 \\
S(4,2)=7 \\
S(4,1)=1\end{array}$ & $\begin{array}{r}219 \\
19 \\
3 \\
3 \\
1\end{array}$ & $\begin{array}{r}219 \\
114 \\
9 \\
12 \\
1\end{array}$ & 355 \\
\hline 5 & $\begin{array}{r}11111 \\
2111 \\
221 \\
311 \\
32 \\
41 \\
5\end{array}$ & $\left.\begin{array}{l}\lambda(5: 0: 5)=1 \\
\lambda(5: 1: 4)=10 \\
\lambda(5: 2: 3)=15 \\
\lambda(5: 3: 3)=10 \\
\lambda(5: 4: 2)=10 \\
\lambda(5: 6: 2)=5 \\
\lambda(5: 10: 1)=1\end{array}\right\}$ & $\left\{\begin{array}{l}S(5,5)=1 \\
S(5,4)=10 \\
S(5,3)=25 \\
S(5,2)=15 \\
S(5,1)=1\end{array}\right.$ & $\begin{array}{r}4231 \\
219 \\
19 \\
19 \\
3 \\
3 \\
1\end{array}$ & $\begin{array}{r}4231 \\
2190 \\
285 \\
190 \\
30 \\
15 \\
1\end{array}$ & 6942 \\
\hline
\end{tabular}

\section{References}

[1] M. Louis Comtet, "Recouvrements, bases de filtre et topologies d'un ensemble fini", C.R. Acad. Sci. Paris Sér. A-B 262 (1966), Al091-Al094.

[2] J.W. Evans, F. Harary and M.S. Lynn, "On the computer enumeration of finite topologies", Comm. ACM 10 (1967), 295-297.

[3] Hansraj Gupta, "Number of topologies on a finite set", Res. BuZl. Panjab Univ. (N.S.) 19 (1968), 231-241.

[4] V. Krishnamurthy, "On the number of topologies on a finite set", Amer. Math. Monthly 73 (1966), 154-157. 
[5] Marlon Rayburn, "On the Borel fields of a finite set", Proc. Amer. Math. Soc. 19 (1968), 885-889.

[6] A. Shafaat, "On the number of topologies definable for a finite set", J. Austral. Math. Soc. 8 (1968), 194-198.

[7] Henry Sharp, Jr., "Quasi-orderings and topologies on finite sets", Proc. Amer. Math. Soc. 17 (1966), 1344-1349.

Department of Mathematics,

Birla Institute of Technology and Science,

Pilani (Rajasthan),

India. 